\title{
Radio Properties of Narrow-Line Seyfert 1 Galaxies
}

\author{
Matthew L. Lister* \\ Department of Physics and Astronomy, Purdue University, USA \\ E-mail: mlisterepurdue.edu
}

\begin{abstract}
The last decade has witnessed a steadily increasing number of observational studies concerning the rare class of radio loud narrow-line Seyfert 1 galaxies, of which several hundred are currently known. According to the current AGN paradigm, the low black hole masses and high accretion rates of narrow-line Seyfert 1 galaxies (NLSy1) should make them unlikely to launch jets, and indeed the vast majority $(\sim 90 \%)$ are very radio weak. The remainder, however, display a wide range of radio power, from $\sim 10^{21-28} \mathrm{~W} \mathrm{~Hz}^{-1}$. In this review I discuss recent radio imaging surveys that suggest there are three main classes of NLSy1, which cannot be easily distinguished by the standard radio-loudness parameter alone: (i) radio-weak NLSy1s without jets, (ii) mildly radio-loud NLSy1s that are a mixture of star-forming and jet-dominant AGN, and (iii) very radio loud NLSy1s with extreme properties similar to powerful jet-dominated blazars. I present updated kinematics information from the MOJAVE survey on six of the latter sources (all detected in gamma-rays by Fermi), indicating high bulk Lorentz factors and small viewing angles in three cases. Studies with the JVLA have shown that the jets of radio loud NLSy1s are likely lowerpower versions of classical radio galaxies, with typical lengths of less than $10 \mathrm{kpc}$, although two very radio-loud NLSy1s have de-projected sizes of several hundred kpc. I discuss the challenges of reconciling the heterogeneous radio properties of NLSy1s with their strict optical line criteria, and near-term prospects for the discovery of larger numbers of radio-loud NLSy1s.
\end{abstract}

Revisiting narrow-line Seyfert 1 galaxies and their place in the Universe - NLS1-2018

9-13 April 2018

Padova Botanical Garden, Italy

${ }^{*}$ Speaker. 


\section{Background}

A longstanding challenge in understanding the AGN phenomenon is being able to use the wealth of information obtained from optical spectroscopy to predict and interpret properties at other wavebands. The current list of known AGN is heavily dominated by optically-selected objects, due to higher achievable étendue of optical surveys compared to those in the radio, IR, X-ray, or $\gamma$-ray, and more efficient spectroscopic followup studies. This has historically led to the introduction of many AGN classes such as narrow-line Seyfert 1 galaxies (NLSy1) that are purely optically-based. As is frequently the case in astronomy, surveys of a particular optical class at other wavelengths can reveal large surprises, and NLSy1s are no exception in this regard.

After the NLSy1 classification criteria were formalized by [1] and [2] in the 1980s, large Xray surveys in the subsequent decade [3] revealed that NLSy1s show steep soft X-ray continua, and are highly variable in the X-ray regime $[4,5]$. Discoveries of NLSy1 grew significantly during the 1990s, as follow-up spectroscopy targeted X-ray sources displaying these properties (Table 1).

In the radio band, the situation proved to be quite different, as hardly any of the approximately 200 known NLSy1s in the year 2000 were known to have associated radio emission. It was only in 2007 that the first cross-correlations of the FIRST VLA $1.4 \mathrm{GHz}$ survey with SDSS revealed $\sim 80$ radio loud NLSy1s $[6,7]$. In carefully selected samples [8] the radio loud fraction was approximately 3-7\%, only half as large as the fraction seen in broad-lined AGN. The low NLSy1 detection rates reflected both the limited sensitivity of the available large radio surveys (e.g., NVSS and FIRST) and the relatively low luminosities of NLSy1s in the radio. As for $\gamma$-rays, the current situation echoes that of the radio band in the early 2000s, with only a very small number of detections by the Fermi LAT instrument, despite its continuous all-sky coverage since 2008. Although $\mathrm{TeV} \gamma$-ray searches have been made with air-shower Cherenkov telescopes [9, 10], no significant NLSy1 detections have been reported to date.

\section{Why study NLSy1s in the radio?}

Given their small numbers and generally weak, hard to study radio emission, it is perhaps surprising to see a large increase in the number of NLSy1 radio studies in the last decade. Community interest in this area has been driven by three main factors:

Imaging capabilities: The radio band offers an unparalleled ability to penetrate the dense gas in the host galaxy nucleus and image the AGN emission in high detail. As described in $\S 4$, the primary instruments in this regard are the JVLA and VLBA, which offer kiloparsec- and pc-scale resolution imaging, respectively, as well as full polarization capability. Both of these instruments have received upgrades since 2010 that significantly improved their flux sensitivity. With the commissioning of ALMA in 2010, we have an additional powerful tool for studying star formation and molecular gas at high resolution in low-luminosity AGN (LLAGN) [11, 12, 13].

Radio Loudness and the AGN Fundamental Plane: NLSy1s appear to defy the current paradigm that powerful jetted outflows are found predominantly in elliptical galaxies with central black hole masses in excess of $10^{8} \mathrm{M}_{\odot}$. Although not many NLSy1 host galaxies have been imaged to date, they appear (like normal Seyferts) to be mainly spirals, but with a higher tendency towards bars [14], pseudo-bulges [15], and denser environments [16]. NLSy1s also lie at the extreme end 
Table 1: Discovery History of NLSy1 Galaxies

\begin{tabular}{||l|cccc||}
\hline & \multicolumn{4}{|c||}{ Year } \\
\hline Category & $\mathbf{1 9 9 0}$ & $\mathbf{2 0 0 0}$ & $\mathbf{2 0 1 0}$ & $\mathbf{2 0 1 8}$ \\
\hline All & 50 & 205 & 2049 & 11472 \\
RL $(10<R<100)$ & 1 & 2 & 54 & 234 \\
VRL $(R>100)$ & 0 & 1 & 29 & 144 \\
GeV $\gamma$-ray & 0 & 0 & 4 & 18 \\
TeV $\gamma$-ray & 0 & 0 & 0 & 0 \\
\hline
\end{tabular}

Notes: the columns indicate the number of known NLSy1s in each category as of the year indicated. RL $=$ radio loud, VRL = very radio loud.

of the Eigenvector 1 distribution (which relates Fe II and $\mathrm{H} \beta$ line widths with the $\mathrm{X}$-ray continuum strength [17]), which is likely a result of their high Eddington ratios. Several AGN studies have shown a strong correlation between the radio loudness parameter $R^{1}$ and black hole mass [18, 19, 20], as well as an anti-correlation between $R$ and accretion rate. Given their low black hole masses and high accretion rates, NLSy1s would not be expected to launch jets, and thus should be radioquiet. This prediction appears to be borne out in low-fraction of radio-detected NLSy1s, however, the general paradigm has difficulty accounting for the sizable number of NLSy1s that are very radio loud (Table 1).

Jetted outflows in NLSy1s: Studies with the VLA and MERLIN in the 1980s revealed that a large fraction of LLAGN generate radio-synchrotron emitting jets [21, 22]. Although LLAGN jets have much lower kinetic power than those of classical radio galaxies, some are capable of escaping the confines of their host galaxy and forming large isotropically-emitting radio lobes [23, 24]. Since they were not expected to launch jets, only two NLSy1 radio imaging studies were carried out prior to $2010[25,26]$, with the results of the latter only partially presented in the proceedings of the 1999 Bad Honnef NLSy1 conference. Both studies indicated that the radio emission was compact ( $<300 \mathrm{pc}$; similar to Seyferts), although a subsequent larger study revealed some NLSy1s with higher radio luminosities, $\simeq 10^{24} \mathrm{~W} \mathrm{~Hz}^{-1}$, and evidence of variable radio emission [26]. As discussed in $\S 4$, direct imaging evidence of jetted outflows in NLSy1s has been obtained only recently $[27,28,29]$. These images clearly show that NLSy1s are capable of generating 'triple' lobe-core-lobe radio jet structures reminiscent of lower-power versions of classical radio galaxies.

\section{Radio Emission From AGN}

The radio loudness parameter $R$ figures prominently in many NLSy1 studies, as it is a readily observable quantity that can be tabulated in large surveys. Its use as a jet/non-jet discriminator can be problematic, however, in the case of NLSy1s since their jet powers tend to be weak, and in most instances, may barely dominate other types of radio emission from the host galaxy. For any given AGN, the total radio output is a combination of (a) any nuclear $(<0.1 \mathrm{kpc})$ or (b) larger scale (> $1 \mathrm{kpc}$ ) jet emission, (c) any emission from a corona/wind on scales $<0.1 \mathrm{kpc}$, and (d) host galaxy star-formation emission, in the form of supernovae and their remnants. High sensitivity

\footnotetext{
${ }^{1} R=S_{\text {radio }} / S_{\text {optical }}$, where $S$ are the flux densities at $5 \mathrm{GHz}$ and $4400 \AA$, respectively
} 
radio studies of nearby optically-selected AGN have shown that at least one of these components is usually present, with truly radio-silent AGN being extremely rare [30].

It can be very difficult or impossible to distinguish between these radio-emitting components in AGN, especially when high resolution imaging is not available. The few available studies with high quality data have shown there is a great deal of variance in the relative strengths of these components for individual AGN, particularly among low-luminosity Seyferts [31, 32, 23]. Because radio emission from AGN jets can attain very high luminosities $\left(\sim 10^{29} \mathrm{~W} \mathrm{~Hz}^{-1}\right.$; [33]) whereas the most powerful known starburst radio luminosities are only $\sim 10^{24} \mathrm{~W} \mathrm{~Hz}^{-1}$, the launching of a jet can potentially increase the radio loudness parameter $R$ of an AGN by several orders of magnitude. Early studies of radio emission in bright optically selected quasar samples [34, 35] showed indications of a bi-modality in the radio loudness distribution at approximately $R \simeq 10$, however, this result has been the subject of intense debate involving selection effects and potential sources of error in the $R$ parameter (see discussion by [30] and [36, 37]). The latter include the effects of obscuration and host galaxy contamination in the optical, uncertainties in k-correcting the $R$ value to the AGN rest frame due to incomplete spectral index information, variability in the (usually non-simultaneously obtained) radio and optical fluxes, and the influence of relativistic beaming on the jet emission. More contemporary studies have found a continuous distribution of $R$ in the AGN population [38, 39]. Indeed, the distributions of $R$, peak radio flux density and luminosity for nearby quasars are all characterized by a single peak that contains a mixture of star-forming and jet-dominated AGN, plus an extended tail comprised of powerful jetted AGN [30]. The boundary at which star-forming AGN begin to dominate the luminosity function is approximately $10^{23} \mathrm{~W} \mathrm{~Hz}^{-1}$ at $5 \mathrm{GHz}$.

The observed $5 \mathrm{GHz}$ radio luminosities of NLSy1s (given the current large survey limits of a few mJy) span a very wide range, from $\sim 10^{21} \mathrm{WHz}^{-1}$ to $10^{28} \mathrm{~W} \mathrm{~Hz}^{-1}$ [29]. The median 1.4 GHz luminosity in the large NLSy1 sample of [40] is $10^{24} \mathrm{~W} \mathrm{~Hz}^{-1}$, right near the star-forming/jet dominant boundary, and this is only for the 5\% of NLSy1s that were detected in the $1.4 \mathrm{GHz}$ FIRST survey. It is clear, therefore, that the radio loudness parameter cannot be used as a reliable jet indicator for the majority of NLSy1s. In particular, the oft-used $R=10$ radio-loud/radio-quiet division, which was based on the original [35] study, appears to fail when applied to large samples of AGN and NLSy1s. The $R$ distribution for the large compilation of NLSy1s in [40] shows a peak at $R=10$, with no evidence of bi-modality among the radio-detected objects (the authors do not include any $R$ upper limit values for NLSy1s without known radio counterparts). Also, there are no clear changes seen in radio morphology [41] or optical variability [40] at $R \simeq 10$, where the influences of a jet should supposedly be visible. It appears clear, therefore, that in all but the most radio loud NLSy1s (i.e., those with $\mathrm{R} \gtrsim 100$ ), other indicators must be used as proof of a jet, such as flux variability, the detection of compact radio emission in VLA or VLBI images, or imaging of radio lobe emission located outside the confines of the host galaxy.

\section{Kiloparsec-scale Radio Properties of NLSy1s}

The general consensus on the radio structures of NLSy1s has been that they are universally steep spectrum and compact, with sizes less than $300 \mathrm{pc}$. The latter number reflects the fact that prior to 2014, all but 6 NLSy1s were apparently unresolved with the VLA, which can achieve a 
typical resolution of $\sim 1^{\prime \prime}$ at frequencies of a few GHz. Apart from the small targeted imaging studies of [25] and [26], which contained 7 and 24 sources, respectively, our knowledge of NLSy1 radio structure up to that time was based almost entirely on the FIRST [42] and NVSS [43] surveys. These surveys, carried out at $1.4 \mathrm{GHz}$, have excellent sky coverage, but relatively poor angular resolution ( 5 " and $45^{\prime \prime}$, respectively), and limited sensitivity ( $\gtrsim 1 \mathrm{mJy}$ for FIRST, and $\gtrsim 2.5 \mathrm{mJy}$ for NVSS) for studying radio quiet NLSy1s, or even the weak, small-scale radio emission associated with radio-loud objects. The surveys differ mainly in their choice of array configurations, with the more extended B-configuration of FIRST offering better angular resolution than NVSS (Dconfiguration) at the expense of limited sensitivity to diffuse lobe emission.

Both FIRST and NVSS were carried out prior to a massive upgrade of the VLA that was completed in early 2013. The new Jansky VLA (JVLA) represents a factor of 10 improvement in sensitivity (capable of attaining a $1 \sigma$ continuum rms level of $<3 \mu \mathrm{Jy}$ in one hour of integration), and offers broadband receivers from $1-50 \mathrm{GHz}$ with the capability of simultaneous in-band spectral index measurements. The JVLA upgrade has enabled several recent studies that have significantly revised our view of NLSy1 radio properties.

The study of [28] obtained JVLA observations of three radio loud NLSy1s with the goal of getting accurate positions for VLBI follow-up observations, and serendipitously discovered triple radio structures in all three. Most of the radio lobes are edge-brightened, with morphologies reminiscent of FR-II radio galaxies, although with much lower radio power and jet lengths of only 10 to $30 \mathrm{kpc}$. However, given the fact that the pc-scale jets in these NLSy1s appear one-sided [44], with the counter-jet likely de-boosted by beaming effects, the de-projected sizes could be as large as $400 \mathrm{kpc}$ (see $\S 6$ ).

A much larger JVLA study of 74 NLSy1s, spanning a wide range of redshift and radio loudness parameter $R$, was undertaken by [29]. Nearly $2 / 3$ of the sample were resolved with the JVLA in its highest resolution configuration at $5 \mathrm{GHz}$ (resolution $\sim 0.3^{\prime \prime}$ ). Interestingly, most of the sources with $R<10$ show faint extended emission around an unresolved core, whereas very few of the flatradio spectrum NLSy1s in the sample display extended emission. This is in contrast to powerful flat-spectrum radio quasars, which frequently show weak diffuse kpc-scale emission consistent with radio lobe structures viewed end-on $[45,46]$. The radio cores of the flat-spectrum NLSy1s have brightness temperature lower limits of $10^{4} \mathrm{~K}$ to $10^{8} \mathrm{~K}$, and many are detected with VLBI (§5), making it unlikely that star formation is the source of their radio emission. The $R<10$ and steep spectrum NLSy1s, on the other hand, have much lower core brightness temperatures, so star formation cannot be ruled out, at least in those sources that do not clearly display one-sided or two-sided jet structures.

A main result of these two studies is that kpc-scale radio emission is now known to be common in NLSy1s, although in the vast majority of cases the overall projected extent is less than $10 \mathrm{kpc}$ (similar to ordinary Seyfert galaxies). There are currently only 16 known examples with radioemitting regions larger than $20 \mathrm{kpc}$, with the largest $(1 \mathrm{H} 0323+342)$ having a projected extent of $140 \mathrm{kpc}$ [47]. This rare group of NLSy1s are all very radio loud $(R>100)$, and $2 / 3$ of them have classical lobe-core-lobe morphology. Fermi has so far detected $\gamma$-ray emission from 6 of these NLSy1s, and the relatively high core dominance in their radio images would suggest that their core emission is likely Doppler boosted by relativistic bulk motion of pc-scale jets viewed at small angles to the line of sight. 


\section{Parsec-scale Radio Properties of NLSy1s}

Very Long Baseline Interferometry (VLBI) is a very efficient tool for detecting jet emission in AGN, since current antenna arrays such as the Very Long Baseline Array (VLBA) lack the short baselines and sensitivity to detect radio emission with brightness temperature below $\sim 10^{5}$ $\mathrm{K}$. The typical frequencies of VLBI observations (1.4 to $86 \mathrm{GHz}$ ) can easily penetrate the dust and gas found in the host galaxy nucleus, offering a clear view of compact jet emission at submilliarcsecond resolution ( $\lesssim 5 \mathrm{pc}$ for the most distant known NLSy $1 \mathrm{~s})$. With multi-frequency and multi-epoch observations, it is possible to precisely identify the location of the central engine, perform spectral ageing studies on the lobe emission, and determine the rates of jet expansion and overall age of the radio jets $[48,49]$.

Several VLBI studies have found that jet activity is common in LLAGN, with their radio cores showing a wide distribution of brightness temperatures, from $10^{5} \mathrm{~K}$ to $10^{8} \mathrm{~K}[50,51,52$, 53]. These studies typically recover only $50 \%$ to $90 \%$ of the total VLA flux density, indicating that most LLAGN have low-surface brightness, diffuse emission on scales up to a few hundred milliarcseconds that is invisible to VLBI $[53,54,55]$. In individual LLAGNs where the radio emission has been studied on different spatial scales (e.g., Mrk 6 [56], Mrk 1239 [57], NGC 6764 [58], NGC 4051 [52], NGC 1068 [59], and NGC 2639 [60]), the radio morphology is often complex and distorted, and it is not clear what fraction of the emission is from poorly collimated jets, winds and/or star formation.

To date only $\sim 40$ NLSy1s have been observed with VLBI, mainly due to the fact that their weak radio fluxes require time-consuming phase-referencing observations, where atmospheric corrections to the measured interferometric visibilities are determined by frequent interleaved scans of a nearby bright calibrator source. Most of the observations have thus focused on high $R$ sources, with the study of 7 NLSy1s by [61] being the only one so far to probe the radio-weak $(R<10)$ regime. The latter authors found that both high- and low- $R$ sources can have core brightness temperatures above $10^{7} \mathrm{~K}$, and can display one-sided or two-sided jet morphology. One-sided morphology was seen in 13 of 15 high $R$ jets by [44], and in 7 of 14 very high $R$ jets by [62], suggesting that the pc-scale emission of highly radio loud NLSy1s is affected by Doppler boosting. A main difference in the pc-scale properties of NLSy1s with respect to other LLAGN is the finding of much higher brightness temperatures (up to $10^{11} \mathrm{~K}$ ) in a small cohort that have been detected at $\mathrm{GeV}$ energies by the Fermi LAT instrument [63, 62, 64].

\section{Jet Kinematics of $\gamma$-ray NLSy1s}

The six brightest known NLSy1s in terms of radio flux density have been the subject of regular monitoring with the VLBA at $15 \mathrm{GHz}$ as part of the MOJAVE program [65]. This program focuses on a flux-limited sample of the brightest radio-loud AGN in the northern sky, as well as radiobright AGN detected by Fermi. All of the several hundred targeted AGN have correlated VLBA flux densities above $0.1 \mathrm{Jy}$ at $15 \mathrm{GHz}$, and $96 \%$ are blazars. Doppler beaming can significantly increase the observed radio flux by factors of up to $\sim 10^{5}$ for highly relativistic jets viewed at small angles to the line of sight [66], so the only non-blazars in high frequency flux-density limited radio AGN surveys tend to be nearby $(z<0.1)$ radio galaxies [67]. Because $\gamma$-ray emission in AGN is 

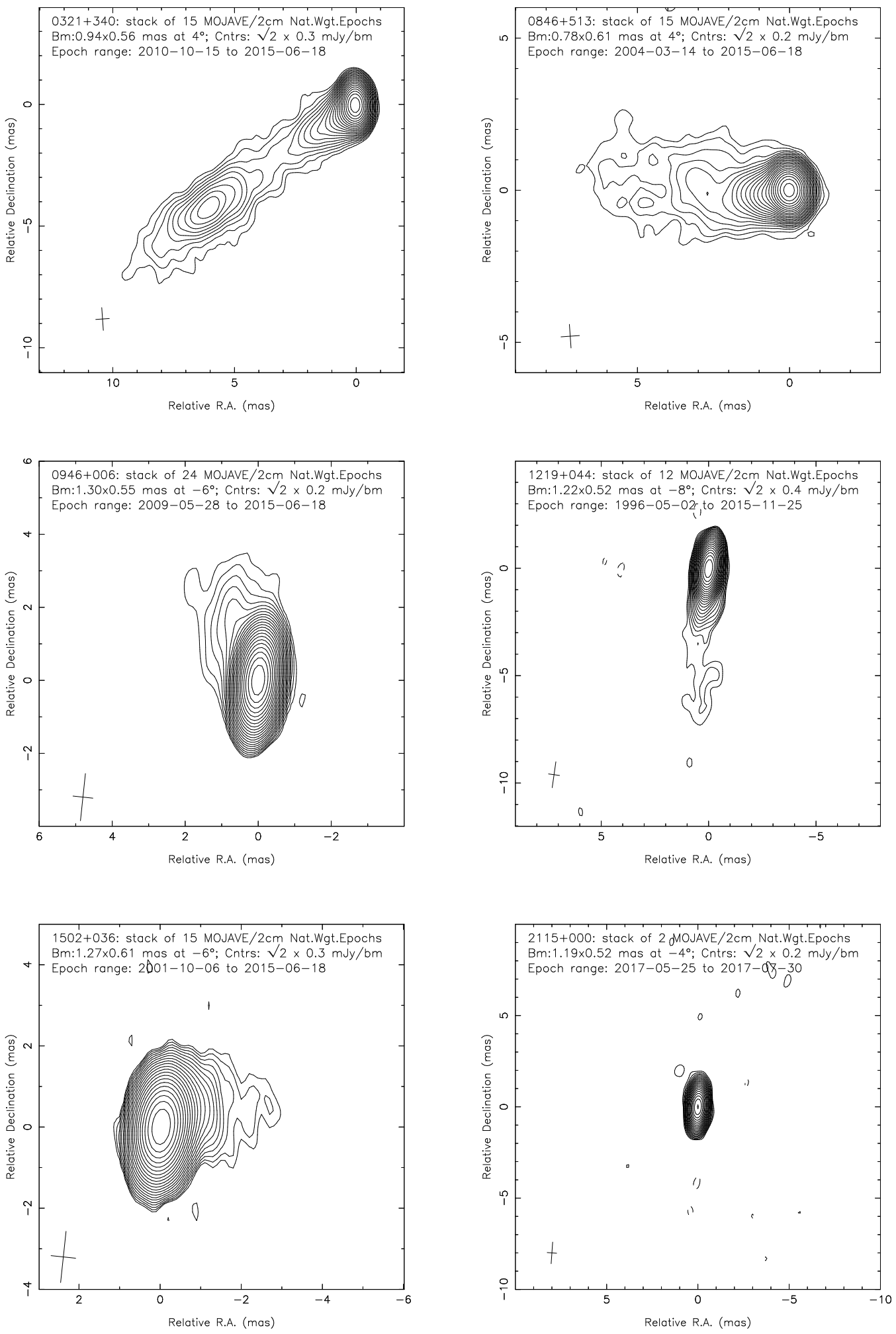

Figure 1: Stacked epoch $15 \mathrm{GHz}$ VLBA images of six $\gamma$-ray loud NLSy1s from the MOJAVE program. 
believed to originate near the bases of powerful relativistic jets, the Fermi LAT AGN catalog suffers from the same Doppler bias, and is also heavily dominated by blazars [68]. Approximately $3 / 4$ of the radio-flux-limited MOJAVE AGN were detected by Fermi in its first four years of operation [69].

The presence of six NLSy1s (all with $R>1000$ ) in the MOJAVE survey (Figure 1) is therefore significant, particularly more so since all six are detected by Fermi. This is strongly indicative of blazar-like properties. The MOJAVE program $[64,70]$ has obtained sufficient VLBA epochs to analyze the jet kinematics of five of these $\gamma$-ray loud NLSy1s; the results are listed in Table 2. Three jets are highly superluminal, while PMN J2118+0013 shows only sub-luminal motion over a 5 year monitoring period, and PKS J1222+0413 shows no measurable motion over a 14 year period.

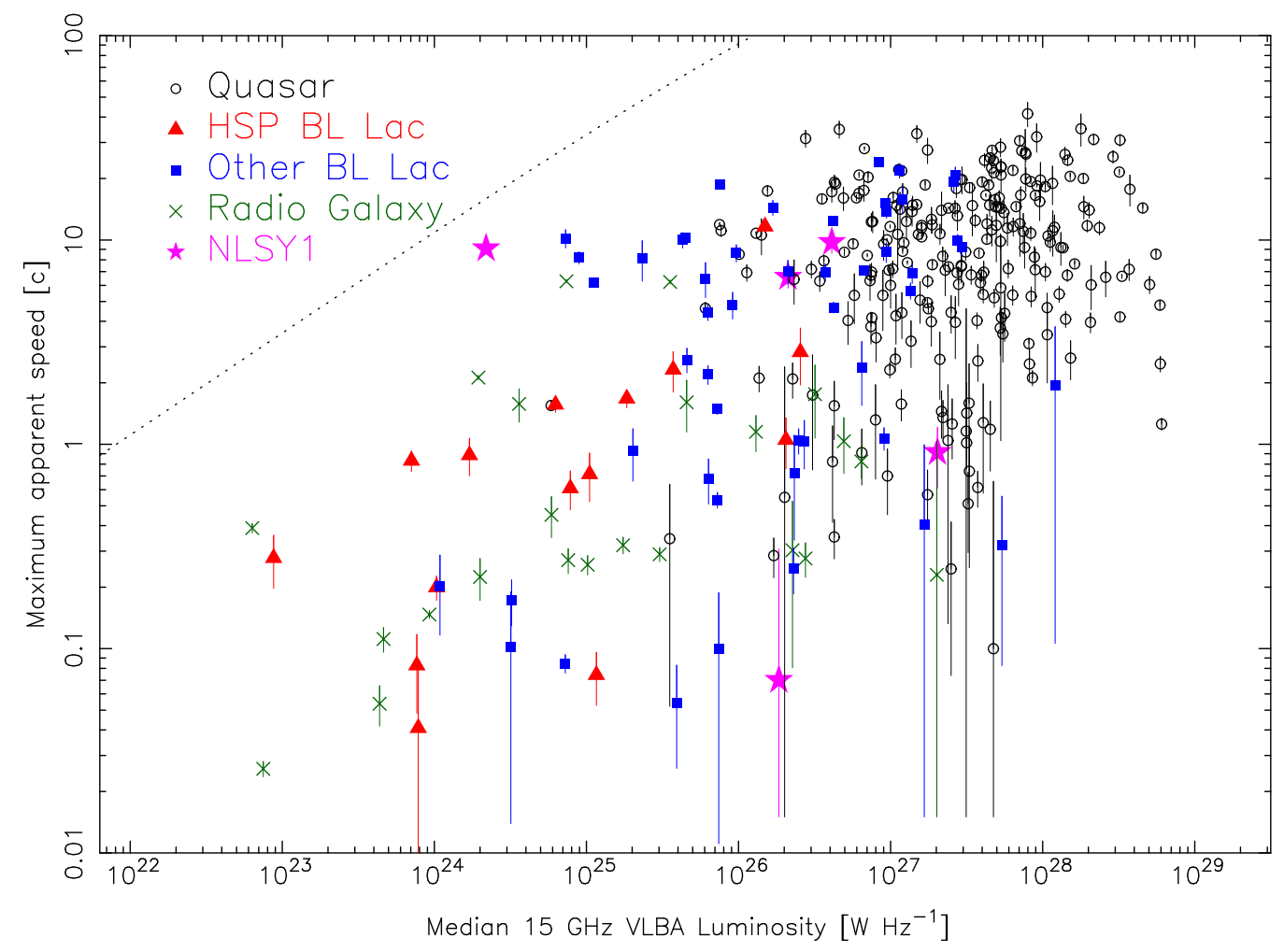

Figure 2: Plot of maximum measured jet speed versus median $15 \mathrm{GHz}$ VLBA radio luminosity for 334 jets in the MOJAVE sample from [64] and [70]. The dotted line indicates the upper limit of the survey.

Figure 2 shows the maximum jet speed plotted against median VLBA $15 \mathrm{GHz}$ luminosity for 334 AGN in the MOJAVE sample. As discussed by [71], the upper envelope to this distribution is a result of the rarity of AGN jets with bulk Lorentz factors above $\sim 30$, and an intrinsic correlation between the true jet speed and un-beamed radio synchrotron luminosity. Two of the $\gamma$-ray NLSy1s (SBS 0846+513 and PMN J0948+0022) lie at the low end of the quasar luminosity distribution, but have similar radio luminosities and speeds to low-synchrotron peaked BL Lac objects. It is worth noting the location of $1 \mathrm{H} 0323+342$ at the extreme upper left edge of the distribution. This NLSy1 displays blazar-like variability at all wavelengths [72], has appreciably high linear polarization in its pc-scale jet [73], and appears to have an atypically large apparent speed $(9.1 \mathrm{c})$ for its radio 
Table 2: Kinematics of $\gamma$-ray Detected NLSy1 Jets

\begin{tabular}{||l|ccccc||}
\hline $\begin{array}{l}\text { Name } \\
(1)\end{array}$ & $\begin{array}{c}\text { Redshift } \\
(2)\end{array}$ & $\begin{array}{c}\beta_{\text {app }}[\mathrm{c}] \\
(3)\end{array}$ & $\begin{array}{c}\theta_{\max }\left[{ }^{\circ}\right] \\
(4)\end{array}$ & $\begin{array}{c}\text { Size }[\mathrm{kpc}] \\
(5)\end{array}$ & $\begin{array}{c}\text { Refs. } \\
(6)\end{array}$ \\
\hline 1H 0323+342 & 0.061 & $9.1 \pm 0.3$ & 13 & 140 & {$[64],[47],[76]$} \\
SBS 0846+513 & 0.585 & $6.6 \pm 0.8$ & 17 & $<3$ & {$[70],[29],[77]$} \\
PMN J0948+0022 & 0.5838 & $9.7 \pm 1.1$ & 12 & 130 & {$[70],[27],[78]$} \\
PKS J1222+0413 & 0.966 & $0.9 \pm 0.3$ & $\ldots$ & 80 & {$[70],[46],[79]$} \\
PKS 1502+036 & 0.4083 & $0.07 \pm 0.2$ & $\ldots$ & 10 & {$[70],[29],[76]$} \\
PMN J2118+0013 & 0.463 & $\ldots$ & $\ldots$ & $<1.5$ & {$[70],[80],[81]$} \\
\hline
\end{tabular}

Columns are as follows: (1) source name; (2) redshift; (3) maximum apparent jet feature speed, in units of the speed of light; (4) maximum jet viewing angle in degrees; (5) largest projected diameter of radio emission in kpc; (6) reference for apparent speed, kpc-scale radio image, and $\gamma$-ray discovery.

luminosity $\left(2 \times 10^{24} \mathrm{WHz}^{-1}\right)$. This could be indicative of a very high intrinsic jet speed and/or high Doppler boosting factor (see, e.g., [74]). The lack of any appreciably measurable jet speed in PKS 1502+036 may simply reflect that it has not ejected any bright features during the five years it was monitored by the MOJAVE program. In fact, there are 67 AGN in the MOJAVE sample which do not have any $>3 \sigma$ speed measurements [70]. In the case of PMN J2118+0013, the VLBA has not yet revealed any detectable emission downstream of its unresolved radio core ${ }^{2}$.

The high superluminal speeds measured for three of these NLSy1s set important constraints on their jet Lorentz factors $\left(\Gamma>v_{\text {app }} / c\right)$ and jet viewing angles $\left(\theta<2 \arctan \left(c / v_{\text {app }}\right)\right)$. As can be seen in Table 1 , the upper limits of $\theta \lesssim 15^{\circ}$ imply de-projected sizes of $\gtrsim 600 \mathrm{kpc}$ for $1 \mathrm{H} 0323+342$ and PMN J0948+0022, which, unless they undergo significant jet bending from pc- to kpc-scales, would put them towards the upper end of the size distribution for powerful radio galaxies [75]. This sets them well apart from the other radio-detected NLSy1s, which tend to have sizes below $\sim 10$ kpc.

\section{Summary}

In the last decade we have seen a flurry of investigations into the radio properties of NLSy1s, with the number of known radio-loud objects growing from just 3 to nearly 400 . These studies have revealed an unprecedented look into the jet activity of these AGN, whose low black hole masses and high accretion rates should strongly disfavor them from launching jets, according to the current AGN paradigm. On the basis of their radio emission, NLSy1s appear to be divided into three broad classes. The vast majority ( $>90 \%$ ) of NLSy1s are optically non-variable [82] and very radio weak, being undetectable above $1 \mathrm{mJy}$ and having radio loudness parameters $R<1$ [40]. Any radio emission in these sources is likely to be from star formation or weakly collimated winds generated by the accretion disk $[83,84]$. The second class contains what are typically termed radio-quiet or mildly radio-loud objects, which have $1<R<100$, and are likely a mixture of starforming dominated and jet-dominated sources. Evidence for the latter comes from the high fraction of NLSy1 that have blazar-like colors in the IR $[84,85]$ and X-ray variability that is much more prevalent than in regular Seyferts $[4,86]$. Their jets appear to be lower-power versions of classical

\footnotetext{
${ }^{2} \mathrm{http} / / /$ www.astro.purdue.edu/MOJAVE
} 
radio galaxies, with lengths less than $10 \mathrm{kpc}$. The rarest category, comprising less than $1 \%$ of NLSy1s, have powerful relativistic jets and display many of the characteristics of flat-spectrum blazars, including high polarization and multi-wavelength variability, and jet sizes up a to few hundred kpc. They can reach radio loudness values in excess of $R=10^{3}$, and 18 have been detected so far in $\gamma$-rays by Fermi.

It remains to be determined why such an apparently heterogeneous collection of AGNs can all meet the rigorous optical spectral line criteria of the NLSy1 class. It has been speculated that orientation effects of (a) geometrically thin broad line region [87],(b) radiation pressure pushing the broad line region to larger distances from the AGN [88], and (c) relativistic beaming of optical jet continuum [89] all may affect observed line strengths, but quantitative statistical assessment of these scenarios awaits data on larger samples of radio-loud NLSy1s. In particular, it will be important to conduct more VLBI studies on all types of LLAGN that span a broad range of $\mathrm{H} \beta$ line width, to avoid possible selection biases in the NLSy1 class [90]. The VLA sky survey (VLASS), currently underway, will provide $2.5^{\prime \prime}$ resolution images, accurate positions, and flux densities from 2 to $5 \mathrm{GHz}$ for over 5,000,000 radio sources in the entire sky north of declination $-40^{\circ}$, down to limiting flux densities of 0.1 to $0.3 \mathrm{mJy}$. The combination of VLASS with large ongoing spectroscopic surveys such as SDSS-BOSS [91] and LAMOST [92] should result in many new NLSy1 candidates. Followup near-IR spectroscopy longward of 10,000 $\AA$ will also be fruitful for discovering NLSy 1 past $z \simeq 0.9$, where the $H \beta$ line is redshifted out of the optical range (e.g.,[93]).

\section{Acknowledgments}

This conference has been organized with the support of the Department of Physics and Astronomy "Galileo Galilei", the University of Padova, the National Institute of Astrophysics INAF, the Padova Planetarium, and the RadioNet consortium. RadioNet has received funding from the European Union's Horizon 2020 research and innovation programme under grant agreement No 730562. The MOJAVE program is supported under NASA-Fermi grants NNX15AU76G and NNX12A087G. The National Radio Astronomy Observatory is a facility of the National Science Foundation operated under cooperative agreement by Associated Universities, Inc.

\section{References}

[1] D. E. Osterbrock and R. W. Pogge, The spectra of narrow-line Seyfert 1 galaxies, ApJ 297 (1985) 166.

[2] R. W. Goodrich, Spectropolarimetry of 'narrow-line' Seyfert 1 galaxies, ApJ 342 (1989) 224.

[3] W. Voges, B. Aschenbach, T. Boller, H. Bräuninger, U. Briel, W. Burkert et al., The ROSAT all-sky survey bright source catalogue, A\&A 349 (1999) 389 [ast ro-ph/9909315].

[4] T. Boller, W. N. Brandt and H. Fink, Soft X-ray properties of narrow-line Seyfert 1 galaxies., A\&A 305 (1996) 53 [astro-ph/9504093].

[5] K. Hayashida, ASCA observations of NLS1s: BH mass estimation from X-ray variability and X-ray spectra, NAR 44 (2000) 419 [astro-ph/ 0005128 ].

[6] D. J. Whalen, S. A. Laurent-Muehleisen, E. C. Moran and R. H. Becker, Optical Properties of Radio-selected Narrow-Line Seyfert 1 Galaxies, AJ 131 (2006) 1948 [astro-ph/ 0601162 ]. 
[7] H. Zhou, T. Wang, W. Yuan, H. Lu, X. Dong, J. Wang et al., A Comprehensive Study of 2000 Narrow Line Seyfert 1 Galaxies from the Sloan Digital Sky Survey. I. The Sample, ApJS 166 (2006) 128 [astro-ph/0603759].

[8] S. Komossa, W. Voges, D. Xu, S. Mathur, H.-M. Adorf, G. Lemson et al., Radio-loud Narrow-Line Type 1 Quasars, AJ 132 (2006) 531 [astro-ph/ 0603680 ].

[9] H. E. S. S. Collaboration, A. Abramowski, F. Aharonian, F. Ait Benkhali, A. G. Akhperjanian, E. Angüner et al., Flux upper limits for 47 AGN observed with H.E.S.S. in 2004-2011, A\&A 564 (2014) A9 [1402.2332].

[10] W. Benbow, Highlights from the VERITAS AGN observation program, in 6th International Symposium on High Energy Gamma-Ray Astronomy, vol. 1792 of American Institute of Physics Conference Series, p. 050001, Jan., 2017, 1708.02374 , DOI.

[11] A. Simionescu, G. Tremblay, N. Werner, R. E. A. Canning, S. W. Allen and J. B. R. Oonk, ALMA observation of the disruption of molecular gas in M87, MNRAS 475 (2018) 3004 [1801. 01264 ].

[12] K. Nyland, T. A. Davis, D. D. Nguyen, A. Seth, J. M. Wrobel, A. Kamble et al., A Multi-wavelength Study of the Turbulent Central Engine of the Low-mass AGN Hosted by NGC 404, ApJ 845 (2017) 50 [1707.02303].

[13] D. Salak, Y. Tomiyasu, N. Nakai and Y. Miyamoto, Multi-line Imaging of the Starburst Galaxy NGC 1808 with ALMA, ArXiv e-prints (2016) [1612.06488].

[14] K. Ohta, K. Aoki, T. Kawaguchi and G. Kiuchi, A Bar Fuels a Supermassive Black Hole?: Host Galaxies of Narrow-Line Seyfert 1 Galaxies, ApJS 169 (2007) 1 [astro-ph/ 0610355 ].

[15] G. Orban de Xivry, R. Davies, M. Schartmann, S. Komossa, A. Marconi, E. Hicks et al., The role of secular evolution in the black hole growth of narrow-line Seyfert 1 galaxies, MNRAS 417 (2011) 2721 [1104.5023].

[16] E. Järvelä, A. Lähteenmäki, H. Lietzen, A. Poudel, P. Heinämäki and M. Einasto, Large-scale environments of narrow-line Seyfert 1 galaxies, A\&A 606 (2017) A9 [1707.01411].

[17] J. W. Sulentic, T. Zwitter, P. Marziani and D. Dultzin-Hacyan, Eigenvector 1: An Optimal Correlation Space for Active Galactic Nuclei, ApJL 536 (2000) L5 [astro-ph/ 0005177 ].

[18] A. Laor, On Black Hole Masses and Radio Loudness in Active Galactic Nuclei, ApJL 543 (2000) L111 [astro-ph/0009192].

[19] J. S. Dunlop, R. J. McLure, M. J. Kukula, S. A. Baum, C. P. O'Dea and D. H. Hughes, Quasars, their host galaxies and their central black holes, MNRAS 340 (2003) 1095 [astro-ph/ 0108397 ].

[20] R. J. McLure and M. J. Jarvis, The relationship between radio luminosity and black hole mass in optically selected quasars, MNRAS 353 (2004) L45 [astro-ph/ 0408203 ].

[21] J. S. Ulvestad and A. S. Wilson, Radio structures of Seyfert galaxies. VI - VLA observations of a nearby sample, ApJ 285 (1984) 439.

[22] S. W. Unger, A. Pedlar, R. V. Booler and B. A. Harrison, Compact radio components in Seyfert galaxies, MNRAS 219 (1986) 387.

[23] S. A. Baum, C. P. O’Dea, D. Dallacassa, A. G. de Bruyn and A. Pedlar, Kiloparsec-Scale Radio Emission in Seyfert Galaxies: Evidence for Starburst-driven Superwinds?, ApJ 419 (1993) 553.

[24] R. D. Baldi, D. R. A. Williams, I. M. McHardy, R. J. Beswick, M. K. Argo, B. T. Dullo et al., LeMMINGs - I. The eMERLIN legacy survey of nearby galaxies. 1.5-GHz parsec-scale radio structures and cores, MNRAS 476 (2018) 3478 [1802 . 02162]. 
[25] J. S. Ulvestad, R. R. J. Antonucci and R. W. Goodrich, Radio properties of narrow-lined Seyfert 1 galaxies, AJ 109 (1995) 81.

[26] E. C. Moran, Radio properties of NLS1s, NAR 44 (2000) 527 [astro-ph/ 0005149 ].

[27] A. Doi, H. Nagira, N. Kawakatu, M. Kino, H. Nagai and K. Asada, Kiloparsec-scale Radio Structures in Narrow-line Seyfert 1 Galaxies, ApJ 760 (2012) 41 [1210.1303].

[28] J. L. Richards and M. L. Lister, Kiloparsec-Scale Jets in Three Radio-Loud Narrow-Line Seyfert 1 Galaxies, ApJL 800 (2015) L8 [1501.05299].

[29] M. Berton, E. Congiu, E. Järvelä, R. Antonucci, P. Kharb, M. L. Lister et al., Radio-emitting narrow-line Seyfert 1 galaxies in the JVLA perspective, ArXiv e-prints (2018) [1801.03519].

[30] K. I. Kellermann, J. J. Condon, A. E. Kimball, R. A. Perley and Ž. Ivezić, Radio-loud and Radio-quiet QSOs, ApJ 831 (2016) 168 [1608.04586].

[31] J. J. Condon, K. I. Kellermann, A. E. Kimball, Ž. Ivezić and R. A. Perley, Active Galactic Nucleus and Starburst Radio Emission from Optically Selected Quasi-stellar Objects, ApJ 768 (2013) 37 [1303.3448].

[32] J. F. Gallimore, D. J. Axon, C. P. O’Dea, S. A. Baum and A. Pedlar, A Survey of Kiloparsec-Scale Radio Outflows in Radio-Quiet Active Galactic Nuclei, AJ 132 (2006) 546 [astro-ph / 0604219 ].

[33] P. Mao, C. M. Urry, E. Marchesini, M. Landoni, F. Massaro and M. Ajello, Radio Luminosity Function of Flat-spectrum Radio Quasars, ApJ 842 (2017) 87.

[34] P. A. Strittmatter, P. Hill, I. I. K. Pauliny-Toth, H. Steppe and A. Witzel, Radio observations of optically selected quasars, A\&A 88 (1980) L12.

[35] K. I. Kellermann, R. Sramek, M. Schmidt, D. B. Shaffer and R. Green, VLA observations of objects in the Palomar Bright Quasar Survey, AJ 98 (1989) 1195.

[36] Y. Terashima and A. S. Wilson, Chandra Snapshot Observations of Low-Luminosity Active Galactic Nuclei with a Compact Radio Source, ApJ 583 (2003) 145 [astro-ph/ 0209607 ].

[37] L. C. Ho and C. Y. Peng, Nuclear Luminosities and Radio Loudness of Seyfert Nuclei, ApJ 555 (2001) 650 [astro-ph/0102502].

[38] S. E. Rafter, D. M. Crenshaw and P. J. Wiita, Radio Properties of Low-Redshift Broad Line Active Galactic Nuclei, AJ 137 (2009) 42 [0809.3977].

[39] J. Singal, V. Petrosian, A. Lawrence and Ł. Stawarz, On the Radio and Optical Luminosity Evolution of Quasars, ApJ 743 (2011) 104 [1101.2930].

[40] S. Rakshit, C. S. Stalin, H. Chand and X.-G. Zhang, A Catalog of Narrow Line Seyfert 1 Galaxies from the Sloan Digital Sky Survey Data Release 12, ApJS 229 (2017) 39 [1704 . 07700].

[41] R. Coziol, H. Andernach, J. P. Torres-Papaqui, R. A. Ortega-Minakata and F. Moreno del Rio, What sparks the radio-loud phase of nearby quasars?, MNRAS 466 (2017) 921 [1612.02655].

[42] D. J. Helfand, R. L. White and R. H. Becker, The Last of FIRST: The Final Catalog and Source Identifications, ApJ 801 (2015) 26 [1501.01555].

[43] J. J. Condon, W. D. Cotton, E. W. Greisen, Q. F. Yin, R. A. Perley, G. B. Taylor et al., The NRAO VLA Sky Survey, AJ 115 (1998) 1693. 
[44] J. L. Richards, M. L. Lister, T. Savolainen, D. C. Homan, M. Kadler, T. Hovatta et al., The parsec-scale structure, kinematics, and polarization of radio-loud narrow-line Seyfert 1 galaxies, in Extragalactic Jets from Every Angle (F. Massaro, C. C. Cheung, E. Lopez and A. Siemiginowska, eds.), vol. 313 of IAU Symposium, pp. 139-142, Mar., 2015, 1411.1058 , DOI.

[45] R. R. J. Antonucci and J. S. Ulvestad, Extended radio emission and the nature of blazars, ApJ 294 (1985) 158.

[46] P. Kharb, M. L. Lister and N. J. Cooper, Extended Radio Emission in MOJAVE Blazars: Challenges to Unification, ApJ 710 (2010) 764 [1001.0731].

[47] S. Antón, I. W. A. Browne and M. J. Marchã, The colour of the narrow line Sy1-blazar 0324+3410, A\&A 490 (2008) 583 [0907.2400].

[48] M. Murgia, Spectral Ages of CSOs and CSS Sources, PASA 20 (2003) 19 [astro-ph/ 0302376 ].

[49] I. Owsianik and J. E. Conway, First detection of hotspot advance in a Compact Symmetric Object. Evidence for a class of very young extragalactic radio sources, A\&A 337 (1998) 69 [astro-ph/9712062].

[50] E. Middelberg, A. L. Roy, N. M. Nagar, T. P. Krichbaum, R. P. Norris, A. S. Wilson et al., Motion and properties of nuclear radio components in Seyfert galaxies seen with VLBI, A\&A 417 (2004) 925 [astro-ph/0402142].

[51] J. M. Anderson, J. S. Ulvestad and L. C. Ho, Low-Luminosity Active Galactic Nuclei at the Highest Resolution: Jets or Accretion Flows?, ApJ 603 (2004) 42 [astro-ph/ 0311303 ].

[52] M. Giroletti and F. Panessa, The Faintest Seyfert Radio Cores Revealed by VLBI, ApJL 706 (2009) L260 [0910.5821].

[53] M. Orienti and M. A. Prieto, Radio structures of the nuclei of nearby Seyfert galaxies and the nature of the missing diffuse emission, MNRAS 401 (2010) 2599 [0911. 0852].

[54] D. V. Lal, P. Shastri and D. C. Gabuzda, Seyfert Galaxies: Nuclear Radio Structure and Unification, ApJ 731 (2011) 68 [1102.3955].

[55] J. Zuther, S. Fischer and A. Eckart, MERLIN $18 \mathrm{~cm}$ observations of intermediate redshift NLS1 galaxies, in Narrow-Line Seyfert 1 Galaxies and their Place in the Universe, p. 64, 2011.

[56] P. Kharb, C. P. O’Dea, S. A. Baum, E. J. M. Colbert and C. Xu, A Radio Study of the Seyfert Galaxy Markarian 6: Implications for Seyfert Life Cycles, ApJ 652 (2006) 177 [astro-ph/ 0607603 ].

[57] A. Doi, An obscured narrow-line Seyfert 1 galaxy candidate, Mrk 1388 with nonthermal jets, PASJ 67 (2015) 15 [1411.3858].

[58] P. Kharb, A. Hota, J. H. Croston, M. J. Hardcastle, C. P. O’Dea, R. P. Kraft et al., Parsec-scale Imaging of the Radio-bubble Seyfert Galaxy NGC 6764, ApJ 723 (2010) 580 [1 009 . 0702].

[59] J. F. Gallimore, S. A. Baum and C. P. O'Dea, The Parsec-Scale Radio Structure of NGC 1068 and the Nature of the Nuclear Radio Source, ApJ 613 (2004) 794 [astro-ph/ 0406062 ].

[60] E. Xanthopoulos, A. H. C. Thean, A. Pedlar and A. M. S. Richards, Linear radio structures in selected Seyfert galaxies, MNRAS 404 (2010) 1966.

[61] A. Doi, K. Asada, K. Fujisawa, H. Nagai, Y. Hagiwara, K. Wajima et al., Very Long Baseline Array Imaging of Parsec-scale Radio Emissions in Nearby Radio-quiet Narrow-line Seyfert 1 Galaxies, ApJ 765 (2013) 69 [1301.4758]. 
[62] M. Gu, Y. Chen, S. Komossa, W. Yuan, Z. Shen, K. Wajima et al., The Radio Properties of Radio-loud Narrow-line Seyfert 1 Galaxies on Parsec Scales, ApJS 221 (2015) 3 [1509.01889].

[63] M. Orienti, F. D’Ammando, M. Giroletti and for the Fermi-LAT Collaboration, High resolution radio observations of gamma-ray emitting Narrow-Line Seyfert 1s, ArXiv e-prints (2012) [1205. 0402 ].

[64] M. L. Lister, M. F. Aller, H. D. Aller, D. C. Homan, K. I. Kellermann, Y. Y. Kovalev et al., MOJAVE: XIII. Parsec-scale AGN Jet Kinematics Analysis Based on 19 years of VLBA Observations at 15 GHz, AJ 152 (2016) 12 [1603.03882].

[65] M. L. Lister, H. D. Aller, M. F. Aller, M. H. Cohen, D. C. Homan, M. Kadler et al., MOJAVE: Monitoring of Jets in Active Galactic Nuclei with VLBA Experiments. V. Multi-Epoch VLBA Images, AJ 137 (2009) 3718 [0812.3947].

[66] R. C. Vermeulen and M. H. Cohen, Superluminal motion statistics and cosmology, ApJ 430 (1994) 467.

[67] M. L. Lister and D. C. Homan, MOJAVE: Monitoring of Jets in Active Galactic Nuclei with VLBA Experiments. I. First-Epoch 15 GHz Linear Polarization Images, AJ 130 (2005) 1389.

[68] M. Ackermann, M. Ajello, W. B. Atwood, L. Baldini, J. Ballet, G. Barbiellini et al., The Third Catalog of Active Galactic Nuclei Detected by the Fermi Large Area Telescope, ApJ 810 (2015) 14 [1501.06054].

[69] M. L. Lister, M. F. Aller, H. D. Aller, T. Hovatta, W. Max-Moerbeck, A. C. S. Readhead et al., Why Have Many of the Brightest Radio-loud Blazars Not Been Detected in Gamma-Rays by Fermi?, ApJL 810 (2015) L9 [1507.05953].

[70] M. L. Lister et al., (2018), in preparation .

[71] M. L. Lister and A. P. Marscher, Statistical Effects of Doppler Beaming and Malmquist Bias on Flux-limited Samples of Compact Radio Sources, ApJ 476 (1997) 572.

[72] V. S. Paliya, S. Sahayanathan, M. L. Parker, A. C. Fabian, C. S. Stalin, A. Anjum et al., The Peculiar Radio-loud Narrow Line Seyfert 1 Galaxy 1H 0323+342, ApJ 789 (2014) 143 [1405. 0715$].$

[73] M. L. Lister, M. F. Aller, H. D. Aller, M. A. Hodge, D. C. Homan, Y. Y. Kovalev et al., MOJAVE. XV. VLBA $15 \mathrm{GHz}$ Total Intensity and Polarization Maps of 437 Parsec-scale AGN Jets from 1996 to 2017, ApJS 234 (2018) 12 [1711.07802].

[74] M. H. Cohen, M. L. Lister, D. C. Homan, M. Kadler, K. I. Kellermann, Y. Y. Kovalev et al., Relativistic Beaming and the Intrinsic Properties of Extragalactic Radio Jets, ApJ 658 (2007) 232 [arXiv:astro-ph/0611642].

[75] A. Saxena, H. J. A. Röttgering and E. E. Rigby, Modelling the luminosities and sizes of radio sources: radio luminosity function at $z=6$, MNRAS 469 (2017) 4083 [1705.03449].

[76] A. A. Abdo, M. Ackermann, M. Ajello, L. Baldini, J. Ballet, G. Barbiellini et al., Radio-Loud Narrow-Line Seyfert 1 as a New Class of Gamma-Ray Active Galactic Nuclei, ApJL 707 (2009) L142 [0911.3485].

[77] F. D’Ammando, M. Orienti, J. Finke, C. M. Raiteri, E. Angelakis, L. Fuhrmann et al., SBS 0846+513: a new $\gamma$-ray-emitting narrow-line Seyfert 1 galaxy, MNRAS 426 (2012) 317 [1207. 30 92].

[78] A. A. Abdo, M. Ackermann, M. Ajello, M. Axelsson, L. Baldini, J. Ballet et al., Fermi/Large Area Telescope Discovery of Gamma-Ray Emission from a Relativistic Jet in the Narrow-Line Quasar PMN J0948+0022, ApJ 699 (2009) 976 [0 905 . 4 558]. 
[79] S. Yao, W. Yuan, H. Zhou, S. Komossa, J. Zhang, E. Qiao et al., Identification of a new $\gamma$-ray-emitting narrow-line Seyfert 1 galaxy, at redshift 1, MNRAS 454 (2015) L16 [1509.03030].

[80] S. E. Healey, R. W. Romani, G. B. Taylor, E. M. Sadler, R. Ricci, T. Murphy et al., CRATES: An All-Sky Survey of Flat-Spectrum Radio Sources, ApJS 171 (2007) 61 [astro-ph/ 0702346 ].

[81] A. A. Abdo, M. Ackermann, M. Ajello, A. Allafort, E. Antolini, W. B. Atwood et al., Fermi Large Area Telescope First Source Catalog, ApJS 188 (2010) 405 [1 002 . 2280].

[82] E. C. Ferrara, The variability characteristics of broad and narrow line Seyfert 1 galaxies, Ph.D. thesis, Georgia State University, 2000.

[83] E. Sani, D. Lutz, G. Risaliti, H. Netzer, L. C. Gallo, B. Trakhtenbrot et al., Enhanced star formation in narrow-line Seyfert 1 active galactic nuclei revealed by Spitzer, MNRAS 403 (2010) 1246 [0908.0280].

[84] A. Caccianiga, S. Antón, L. Ballo, L. Foschini, T. Maccacaro, R. Della Ceca et al., WISE colours and star formation in the host galaxies of radio-loud narrow-line Seyfert 1, MNRAS 451 (2015) 1795 [1504.07068].

[85] E. Järvelä, A. Lähteenmäki and J. León-Tavares, Statistical multifrequency study of narrow-line Seyfert 1 galaxies, A\&A 573 (2015) A76 [1410.2743].

[86] I. M. McHardy, E. Koerding, C. Knigge, P. Uttley and R. P. Fender, Active galactic nuclei as scaled-up Galactic black holes, Nature 444 (2006) 730 [astro-ph/ 0612273 ].

[87] R. Decarli, M. Dotti, M. Fontana and F. Haardt, Are the black hole masses in narrow-line Seyfert 1 galaxies actually small?, MNRAS 386 (2008) L15 [0801 . 4560].

[88] A. Marconi, D. J. Axon, R. Maiolino, T. Nagao, G. Pastorini, P. Pietrini et al., The Effect of Radiation Pressure on Virial Black Hole Mass Estimates and the Case of Narrow-Line Seyfert 1 Galaxies, ApJ 678 (2008) 693 [0802.2021].

[89] G. Ghisellini, L. Maraschi and F. Tavecchio, The Fermi blazars' divide, MNRAS 396 (2009) L105 [0903.2043].

[90] M. Valencia-S., J. Zuther, A. Eckart, S. Smajic, C. Iserlohe, M. Garcia-Marin et al., Are Narrow Line Seyfert 1 galaxies a special class of Active Galactic Nuclei?, in Proceedings of Nuclei of Seyfert galaxies and QSOs - Central engine \& conditions of star formation (Seyfert 2012). 6-8 November, 2012. Max-Planck-Insitut für Radioastronomie (MPIfR), Bonn, Germany., p. 17, 2012, 1305. 3273.

[91] K. S. Dawson, D. J. Schlegel, C. P. Ahn, S. F. Anderson, É. Aubourg, S. Bailey et al., The Baryon Oscillation Spectroscopic Survey of SDSS-III, AJ 145 (2013) 10 [1208 . 0022 ].

[92] X. Y. Dong, X.-B. Wu, Y. L. Ai, J. Y. Yang, Q. Yang, F. Wang et al., The Large Sky Area Multi-Object Fibre Spectroscopic Telescope (LAMOST) Quasar Survey: Quasar Properties from Data Release Two and Three, AJ 155 (2018) 189 [1803.03063].

[93] M. Berton, L. Foschini, A. Caccianiga, S. Ciroi, E. Congiu, V. Cracco et al., An orientation-based unification of young jetted active galactic nuclei: the case of $3 C 286$, Frontiers in Astronomy and Space Sciences 4 (2017) 8 [1705.07905]. 